\title{
Supersymmetric electroweak cosmic strings
}

\author{
José D. Edelstein* \\ Departament d'Estructura i Constituents de la Matèria, Facultat de Física, Universitat de Barcelona, Diagonal 647, \\ 08028 Barcelona, Spain \\ Carlos Núñez ${ }^{\dagger}$ \\ Departamento de Física, Universidad Nacional de La Plata, C.C. 67, (1900) La Plata, Argentina
}

(Received 30 April 1996)

\begin{abstract}
We study the connection between $N=2$ supersymmetry and a topological bound in a two-Higgs-doublet system with a $\mathrm{SU}(2) \times \mathrm{U}(1)_{Y} \times \mathrm{U}(1)_{Y^{\prime}}$ gauge group. We derive the Bogomol'nyi equations from supersymmetry considerations showing that they hold provided certain conditions on the coupling constants, which are a consequence of the huge symmetry of the theory, are satisfied. Their solutions, which can be interpreted as electroweak cosmic strings breaking one-half of the supersymmetries of the theory, are studied. Certain interesting limiting cases of our model which have recently been considered in the literature are finally analyzed. [S0556-2821(97)04706-1]
\end{abstract}

PACS number(s): 11.27.+d, 12.60.Jv, 98.80.Cq

\section{INTRODUCTION}

Supersymmetric (SUSY) grand unified theories (GUTs) have attracted much attention in connection with the hierarchy problem in possible unified theories of strong and electroweak interactions [1,2]. In view of the requirement of electroweak symmetry breaking, these models necessitate an enrichment of the Higgs sector [3], thereby raising many interesting questions both from the classical and the quantum point of view. In particular, many authors have explored the existence of stable electroweak vortex solutions in a variety of multi-Higgs-doublet systems [4-6] that mimic the bosonic sector of SUSY GUTs, in correspondence with what happens in the Abelian Higgs model [7]. It has also been argued that GUT cosmic strings may exhibit superconducting properties [8], and this fact has recently stimulated the study of several multi-Higgs-doublet models describing many interesting phenomena $[9,10]$.

Vortices emerging as finite energy solutions of gauge theories can be usually shown to satisfy a topological bound for the energy, the so-called Bogomol'nyi bound [11,12]. Originally, these bounds were obtained by writing the energy of the configuration (per unit length) as a sum of squares plus a topological term. There exists another approach to study the Bogomol'nyi relationships (i.e., Bogomol'nyi bound and equations) which exploits the huge symmetry of the theory: It is based on the observation that Bogomol'nyi bounds reflect the presence of an extended supersymmetric structure [13-16]. In particular, for gauge theories with spontaneous symmetry breaking and a topological charge, admitting of an $N=1$ supersymmetric version, it was shown that the $N=2$ supersymmetric extension, which requires certain conditions on coupling constants, has a central charge coinciding with the topological charge $[15,16]$. Having originated from the

\footnotetext{
* On leave from Universidad Nacional de La Plata. Electronic address: edels@ecm.ub.es

†Electronic address: nunez@venus.fisica.unlp.edu.ar
}

supercharge algebra, the bound is expected to be quantum mechanically exact.

Since multi-Higgs-doublet models can be understood to be motivated by SUSY GUTs, supersymmetry provides a natural framework for studying Bogomol'nyi bounds. In fact, we have recently considered in Ref. [17] the supersymmetric extension of the two-Higgs-doublet model first presented in [6], showing that Bogomol'nyi equations are a direct consequence of the underlying $N=2$ supersymmetry of the model. We shall study in this paper a supersymmetric formulation of a $\mathrm{SU}(2) \times \mathrm{U}(1)_{Y} \times \mathrm{U}(1)_{Y^{\prime}}$ model with two Higgs doublets which is a generalization of the one analyzed in [17]. The theory has the same gauge group structure as that of supersymmetric extensions of the Weinberg-Salam model that arise as low energy limits of $\mathrm{E}_{6}$-based grand unified theories or $\mathrm{E}_{8} \times \mathrm{E}_{8}$ superstring theories compactified on a Calabi-Yau manifold with a $\mathrm{SU}(3)$ holonomy. This gauge group was recently considered in Ref. [5] for the study of electroweak strings and, generically, the inclusion of an extra U(1) factor in multi-Higgs-doublet systems has been also taken into account in a variety of models exhibiting cosmic strings [8,9]. In spite of being a simplified model (in the sense that its Higgs structure is not so rich as that of grand unified theories), it can be seen as the simplest extension of the standard model necessary for having the Bogomol'nyi equations. We show that the Bogomol'nyi bound of the model, as well as the Bogomol'nyi equations, is a direct consequence of the requirement of $N=2$ supersymmetry imposed on the theory. We also show explicitly that, as a necessary condition for achieving the $N=2$ model, certain relations between coupling constants must be satisfied. These "critical values" of the coupling constants have physical relevance; e.g., the required relation between coupling constants in the Abelian Higgs model corresponds to the limit between type-I and type-II superconductivity in the relativistic Ginsburg-Landau model [16]. We discuss the solutions of the Bogomol'nyi equations and present some interesting limiting cases.

The paper is organized as follows. In Sec. II, we present the $\mathrm{SU}(2) \times \mathrm{U}(1)_{Y} \times \mathrm{U}(1)_{Y^{\prime}}$ two-Higgs-doublet model in 
$2+1$ dimensions admitting of nontrivial topological configurations and we embed it in an $N=1$ supersymmetric theory. We show that the $N=2$ supersymmetric extension can be obtained provided some relations between coupling constants, analogous to the critical relation appearing in the Abelian Higgs model [16], hold. In Sec. III, we construct the $N=2$ supercharges of the theory, and compute the corresponding supersymmetry algebra. After static configurations are considered, and restricting our calculations to the bosonic sector, we find that the Bogomol'nyi relationships appear as a direct algebraic consequence. This fact clarifies in our theory the model-independent analysis established in Ref. [15]. We further comment on some interesting features of the classical field solutions saturating the Bogomol'nyi bound. These could be interpreted as electroweak cosmic strings breaking half of the supersymmetries of the theory.

Our approach being general and systematic, we finally consider in Sec. IV some limiting cases describing various models which have been recently considered in the literature.

\section{II. $\mathrm{SU}(2) \times \mathrm{U}(1)_{Y} \times \mathrm{U}(1)_{Y^{\prime}} N=2$ SUPERSYMMETRIC} MODEL

We start with a $\mathrm{SU}(2) \times \mathrm{U}(1)_{Y} \times \mathrm{U}(1)_{Y^{\prime}}$ gauge theory, which is described by the action

$$
\begin{aligned}
S= & \int d^{3} x\left[-\frac{1}{4} \vec{W}_{\mu \nu} \cdot \vec{W}^{\mu \nu}-\frac{1}{4} F_{\mu \nu} F^{\mu \nu}-\frac{1}{4} G_{\mu \nu} G^{\mu \nu}\right. \\
& +\frac{1}{2}\left|\mathcal{D}_{\mu}^{(1)} \Phi_{(1)}\right|^{2}+\frac{1}{2}\left|\mathcal{D}_{\mu}^{(2)} \Phi_{(2)}\right|^{2}+\frac{1}{2}\left(\partial_{\mu} A\right)^{2}+\frac{1}{2}\left(\partial_{\mu} B\right)^{2} \\
& \left.+\frac{1}{2}\left(D_{\mu} \vec{W}\right)^{2}-V\left(\Phi_{(1)}, \Phi_{(2)}, A, B, \vec{W}\right)\right]
\end{aligned}
$$

where $\Phi_{(1)}$ and $\Phi_{(2)}$ are a couple of Higgs doublets under the $\mathrm{SU}(2)$ part of the gauge group, $A$ and $B$ are real scalar fields, and $\vec{W}=W^{a} \tau^{a}$ is a real scalar in the adjoint representation of SU(2). The metric is choosen to be $g^{\mu \nu}=(+--)$ and the specific form of the potential will be determined below. The strength fields can be written in terms of gauge fields as

$$
F_{\mu \nu}=\partial_{\mu} A_{\nu}-\partial_{\nu} A_{\mu}, \quad G_{\mu \nu}=\partial_{\mu} B_{\nu}-\partial_{\nu} B_{\mu},
$$

and

$$
W_{\mu \nu}^{a}=\partial_{\mu} W_{\nu}^{a}-\partial_{\nu} W_{\mu}^{a}+g f_{a b c} W_{\mu}^{b} W_{\nu}^{c},
$$

while the covariant derivative is defined as

$$
\mathcal{D}_{\mu}^{(q)} \Phi_{(q)}=\left(\partial_{\mu}+\frac{i}{2} g W_{\mu}^{a} \tau^{a}+\frac{i}{2} \alpha_{(q)} A_{\mu}+\frac{i}{2} \beta_{(q)} B_{\mu}\right) \Phi_{(q)}
$$

$$
q=1,2,
$$

where $g$ is the $\mathrm{SU}(2)$ coupling constant while $\alpha_{(q)}$ and $\beta_{(q)}$ represents the different couplings of $\Phi_{(q)}$ with $A_{\mu}$ and $B_{\mu}$.

A minimal $N=1$ supersymmetric extension of this model is given by an action which in superspace reads

$$
\begin{aligned}
\mathcal{S}_{N=1}= & \frac{1}{2} \int d^{3} x d^{2} \theta\left\{\overline{\Omega_{A}} \Omega_{A}+\overline{\Omega_{B}} \Omega_{B}+\overline{\Omega_{\vec{W}}^{a}} \Omega_{\vec{W}}^{a}-\overline{\mathcal{D} \mathcal{A}} \mathcal{D} \mathcal{A}-\overline{\mathcal{D B}} \mathcal{D B}-\overline{\mathcal{D} W^{a}} \mathcal{D} \mathcal{W}^{a}+\xi_{1} \mathcal{A}+\xi_{2} \mathcal{B}\right. \\
& \left.+\frac{1}{2} \sum_{q=1}^{2}\left[\overline{\left(\overline{\nabla^{(q)}} \Upsilon_{(q)}\right)^{a}}\left(\nabla^{(q)} \Upsilon_{(q)}\right)^{a}+i \Upsilon_{(q)}^{\dagger}\left(\sqrt{2 \lambda_{1}^{(q)}} \mathcal{A}+\sqrt{2 \lambda_{2}^{(q)}} \mathcal{B}+\sqrt{2 \lambda_{3}} \mathcal{W}^{a} \tau^{a}\right) \Upsilon_{(q)}\right]\right\},
\end{aligned}
$$

where

$$
\nabla^{(q)} \Upsilon_{(q)}=\left(\mathcal{D}+\frac{i}{2} g \Gamma_{\vec{W}}+\frac{i}{2} \alpha_{(q)} \Gamma_{A}+\frac{i}{2} \beta_{(q)} \Gamma_{B}\right) \Upsilon_{(q)}
$$

This action is built from a couple of complex doublet superfields $\Upsilon_{(q)} \equiv\left(\Phi_{(q)}, \Psi_{(q)}, F_{(q)}\right)$, three real scalar superfields $\mathcal{A} \equiv\left(A, \chi_{A}, a\right), \mathcal{B} \equiv\left(B, \chi_{B}, b\right)$, and $\mathcal{W} \equiv\left(W^{a}, \chi_{\vec{W}}^{a}, w^{a}\right) \tau^{a}$, and three spinor gauge superfields which in the Wess-Zumino gauge $\operatorname{read} \Gamma_{A} \equiv\left(A_{\mu}, \rho_{A}\right), \Gamma_{B} \equiv\left(B_{\mu}, \rho_{B}\right)$, and $\Gamma_{\vec{W}} \equiv \Gamma_{\vec{W}}^{a} \tau^{a}=\left(W_{\mu}^{a}, \lambda^{a}\right) \tau^{a} . \Omega_{A}, \Omega_{B}$, and $\Omega_{\vec{W}}^{a}$, are the corresponding superfield strengths. Concerning $\lambda_{1}^{(q)}, \lambda_{2}^{(q)}, \lambda_{3}, \xi_{1}$, and $\xi_{2}$, they are real constants whose significance will be clear below. It must be stressed that fermions $\rho_{A}, \rho_{B}, \chi_{A}$, and $\chi_{B}$ are Majorana, $\chi_{\vec{W}}^{a} \tau^{a}$ and $\lambda^{a} \tau^{a}$ are Majorana spinors in the adjoint representation of SU(2), while the Higgsino doublets $\Psi_{(q)}$ are Dirac spinors. $A$ and $B$ are real scalar fields and $W^{a} \tau^{a}$ is a Hermitian field in the adjoint representation of $\mathrm{SU}(2)$. Here $F_{(q)}, a, b$, and $w^{a}$ are auxiliary fields which will be eliminated in what follows using their equations of motion. Finally, $\mathcal{D}$ is the usual supercovariant derivative:

$$
\mathcal{D}=\partial \bar{\theta}+i \bar{\theta} \gamma^{\mu} \partial_{\mu}
$$

with the $\gamma$ matrices being represented by $\gamma^{0}=\tau^{3}, \gamma^{1}=i \tau^{1}$, and $\gamma^{2}=-i \tau^{2}$.

Written in components, action (5) takes the form 


$$
\mathcal{S}=S+\mathcal{S}_{\text {fer }}
$$

where

$$
\begin{aligned}
\mathcal{S}_{\text {fer }}= & \frac{1}{2} \int d^{3} x\left[\frac{i}{2} \sum_{q=1}^{2} \bar{\Psi}_{(q)} \mathbb{D}^{(q)} \Psi_{(q)}+\frac{i}{2} \overline{\Lambda^{a}}(\not D \Lambda)^{a}+\frac{i}{2} \bar{\chi}_{\vec{W}}^{a}\left(\not D \chi_{\vec{W}}\right)^{a}+\frac{i}{2} \overline{\chi_{A}} b \chi_{A}+\frac{i}{2} \overline{\rho_{A}} b \rho_{A}+\frac{i}{2} \overline{\chi_{B}} \partial \chi_{B}+\frac{i}{2} \overline{\rho_{B}} \partial \rho_{B}-g f^{a b c} W^{a} \overline{\Lambda^{b}} \chi_{\vec{W}}^{c}\right. \\
& +\sum_{q=1}^{2}\left[i g \bar{\Psi}_{(q)} \Lambda^{a} \tau^{a} \Phi_{(q)}-\sqrt{8 \lambda_{3}} \bar{\Psi}_{(q)} \chi_{\vec{W}}^{a} \tau^{a} \Phi_{(q)}+i \alpha_{(q)} \bar{\Psi}_{(q)} \rho_{A} \Phi_{(q)}-\sqrt{8 \lambda_{1}^{(q)}} \bar{\Psi}_{(q)} \chi_{A} \Phi_{(q)}+i \beta_{(q)} \bar{\Psi}_{(q)} \rho_{B} \Phi_{(q)}\right. \\
& \left.\left.-\sqrt{8 \lambda_{2}^{(q)}} \bar{\Psi}_{(q)} \chi_{B} \Phi_{(q)}\right]-\sum_{q=1}^{2}\left[\bar{\Psi}_{(q)}\left(\sqrt{2 \lambda_{1}^{(q)}} A+\sqrt{2 \lambda_{2}^{(q)}} B+\sqrt{2 \lambda_{3}} W^{a} \tau^{a}\right) \Psi_{(q)}\right]+\text { H.c. }\right]
\end{aligned}
$$

The potential in Eq. (8) reads

$$
\begin{aligned}
V\left(\Phi_{(1)}, \Phi_{(2)}, A, B, \vec{W}\right)= & \left(\sum_{q=1}^{2} \sqrt{\lambda_{1}^{(q)}} \Phi_{(q)}^{\dagger} \Phi_{(q)}-\frac{\xi_{1}}{\sqrt{2}}\right)^{2}+\left(\sum_{q=1}^{2} \sqrt{\lambda_{2}^{(q)}} \Phi_{(q)}^{\dagger} \Phi_{(q)}-\frac{\xi_{2}}{\sqrt{2}}\right)^{2} \\
& +2 \sum_{q=1}^{2}\left|\left(\sqrt{\lambda_{1}^{(q)}} A+\sqrt{\lambda_{2}^{(q)}} B+\sqrt{\lambda_{3}} \vec{W}\right) \Phi_{(q)}\right|^{2}+\lambda_{3}\left(\sum_{q=1}^{2} \Phi_{(q)}^{\dagger} \tau^{a} \Phi_{(q)}\right)^{2}
\end{aligned}
$$

The preceding action (8) is invariant under the following set of $N=1$ supersymmetry transformations with parameter $\eta$ :

$$
\begin{aligned}
& \delta W_{\mu}^{a}=-i \overline{\eta \gamma}_{\mu} \Lambda^{a}, \quad \delta \Lambda^{a}=-i^{\star} W^{\lambda a} \gamma_{\lambda} \eta, \quad \delta W^{a}=\overline{\eta \chi}_{\vec{W}}^{a}, \quad \delta A=\overline{\eta \chi}_{A}, \quad \delta B=\overline{\eta \chi}_{B}, \\
& \delta A_{\mu}=-i \overline{\eta \gamma}_{\mu} \rho_{A}, \quad \delta \rho_{A}=-i^{\star} F^{\lambda} \gamma_{\lambda} \eta, \quad \delta B_{\mu}=-i \overline{\eta \gamma}_{\mu} \rho_{B}, \quad \delta \rho_{B}=-i^{\star} G^{\lambda} \gamma_{\lambda} \eta \\
& \delta \Phi_{(q)}=\overline{\eta \Psi}_{(q)}, \quad \delta \chi_{\vec{W}}^{a}=-\left[\sum_{q=1}^{2} \sqrt{2 \lambda_{3}} \Phi_{(q)}^{\dagger} \tau^{a} \Phi_{(q)}+i(\not D W)^{a}\right] \eta, \\
& \delta \chi_{A}=-\left[\sum_{q=1}^{2} \sqrt{2 \lambda_{1}^{(q)}} \Phi_{(q)}^{\dagger} \Phi_{(q)}-\xi_{1}+i b A\right] \eta, \\
& \delta \chi_{B}=-\left[\sum_{q=1}^{2} \sqrt{2 \lambda_{2}^{(q)}} \Phi_{(q)}^{\dagger} \Phi_{(q)}-\xi_{2}+i b B\right] \eta, \\
& \delta \Psi_{(q)}=\left[-i \gamma^{\mu} \mathcal{D}_{\mu}^{(q)} \Phi_{(q)}-\left(\sqrt{8 \lambda_{1}^{(q)}} A+\sqrt{8 \lambda_{2}^{(q)}} B+\sqrt{8 \lambda_{3}} W^{a} \tau^{a}\right) \Phi_{(q)}\right] \eta,
\end{aligned}
$$

where ${ }^{\star} W^{\lambda a},{ }^{\star} F^{\lambda}$, and ${ }^{\star} G^{\lambda}$ are the dual field strengths,

$$
{ }^{\star} W^{\lambda a}=\frac{1}{2} \epsilon^{\mu \nu \lambda} W_{\mu \nu}{ }^{a}, \quad{ }^{\star} F^{\lambda}=\frac{1}{2} \epsilon^{\mu \nu \lambda} F_{\mu \nu}, \text { and }{ }^{\star} G^{\lambda}=\frac{1}{2} \epsilon^{\mu \nu \lambda} G_{\mu \nu} .
$$

Now, in order to impose the $N=2$ supersymmetric invariance of the theory, we can consider transformations with a complex parameter $\eta_{c}$ (an infinitesimal Dirac spinor), since this implies the existence of two supersymmetries [14]. Here $\rho_{A}, \rho_{B}$, $\chi_{A}$, and $\chi_{B}$ being real spinors, we combine them into Dirac fermions $\Sigma_{A}$ and $\Sigma_{B}$ given by

$$
\Sigma_{A} \equiv \chi_{A}-i \rho_{A}, \quad \Sigma_{B} \equiv \chi_{B}-i \rho_{B}
$$

We also construct a Dirac fermion $\Xi^{a}$ in the adjoint representation of $\mathrm{SU}(2)$ from $\Lambda^{a}$ and $\chi_{\vec{W}}^{a}$ :

$$
\Xi^{a} \equiv \chi_{\vec{W}}^{a}-i \Lambda^{a}
$$

Using the fermion field redefinitions (13) and (14), the fermionic contribution to the action in Eq. (9) can be rearranged into the form 


$$
\begin{aligned}
\mathcal{S}_{\mathrm{fer}}= & \frac{1}{2} \int d^{3} x\left[\frac{i}{2} \sum_{q=1}^{2} \bar{\Psi}_{(q)} \mathcal{D}^{(q)} \Psi_{(q)}+\frac{i}{2} \bar{\Xi}^{a}(\not D \Xi)^{a}+\frac{i}{2} \bar{\Sigma}_{A} b \Sigma_{A}+\frac{i}{2} \bar{\Sigma}_{B} b \Sigma_{B}-i g f^{a b c} W^{a} \bar{\Xi}^{b} \Xi^{c}-\sum_{q=1}^{2}\left(\overline { \Psi } _ { ( q ) } \left(\sqrt{2 \lambda_{1}^{(q)}} A+\sqrt{2 \lambda_{2}^{(q)}} B\right.\right.\right. \\
& \left.+\sqrt{2 \lambda_{3}} W^{a} \tau^{a}\right) \Psi_{(q)}+\frac{g-\sqrt{8 \lambda_{3}}}{2} \bar{\Psi}_{(q)} \widetilde{\Xi}^{a} \tau^{a} \Phi_{(q)}-\frac{g+\sqrt{8 \lambda_{3}}}{2} \bar{\Psi}_{(q)} \Xi^{a} \tau^{a} \Phi_{(q)}+\frac{\alpha_{(q)}-\sqrt{8 \lambda_{1}^{(q)}}}{2} \bar{\Psi}_{(q)} \widetilde{\Sigma}_{A} \Phi_{(q)} \\
& \left.\left.-\frac{\alpha_{(q)}+\sqrt{8 \lambda_{1}^{(q)}}}{2} \bar{\Psi}_{(q)} \Sigma_{A} \Phi_{(q)}+\frac{\beta_{(q)}-\sqrt{8 \lambda_{2}^{(q)}}}{2} \bar{\Psi}_{(q)} \widetilde{\Sigma}_{B} \Phi_{(q)}-\frac{\beta_{(q)}+\sqrt{8 \lambda_{2}^{(q)}}}{2} \bar{\Psi}_{(q)} \Sigma_{B} \Phi_{(q)}\right)+ \text { H.c. }\right] .
\end{aligned}
$$

Here $\widetilde{\Xi}^{a}, \widetilde{\Sigma}_{A}$, and $\widetilde{\Sigma}_{B}$ are the charge conjugates (the complex conjugates) of $\Xi^{a}, \Sigma_{A}$, and $\Sigma_{B}$, respectively.

We shall be mainly interested in purely bosonic backgrounds where all fermion fields vanish. Given a functional $\mathcal{F}$ depending both on bosonic and fermionic fields, it will then be convenient to define $\mathcal{F} \mid$ for

$$
\mathcal{F}|\equiv \mathcal{F}|_{\Psi_{(q)}, \Sigma_{A}, \Sigma_{B}, \Xi^{a}=0}
$$

Under condition (16) the only nonvanishing supersymmetric transformations (11) are those corresponding to fermionic fields:

$$
\begin{gathered}
\delta_{\eta} \Xi^{a} \mid=-\left[{ }^{\star} W^{\lambda a} \gamma_{\lambda}+\sum_{q=1}^{2} \sqrt{2 \lambda_{3}} \Phi_{(q)}^{\dagger} \tau^{a} \Phi_{(q)}+i(\not D W)^{a}\right] \eta \\
\delta_{\eta} \Sigma_{A} \mid=-\left[{ }^{\star} F^{\lambda} \gamma_{\lambda}+\sum_{q=1}^{2} \sqrt{2 \lambda_{1}^{(q)}} \Phi_{(q)}^{\dagger} \Phi_{(q)}-\xi_{1}+i \not \partial A\right] \eta \\
\delta_{\eta} \Sigma_{B} \mid=-\left[{ }^{\star} G^{\lambda} \gamma_{\lambda}+\sum_{q=1}^{2} \sqrt{2 \lambda_{2}^{(q)}} \Phi_{(q)}^{\dagger} \Phi_{(q)}-\xi_{2}+i \partial B B\right] \\
\delta_{\eta} \Psi_{(q)} \mid=\left[-i \gamma^{\mu} \mathcal{D}_{\mu}^{(q)} \Phi_{(q)}-\left(\sqrt{8 \lambda_{1}^{(q)}} A+\sqrt{8 \lambda_{2}^{(q)}} B\right.\right. \\
\left.\left.+\sqrt{8 \lambda_{3}} W^{a} \tau^{a}\right) \Phi_{(q)}\right] \eta .
\end{gathered}
$$

Now, the transformations (17)-(20) with complex parameter $\eta_{c}=\eta e^{-i \alpha}$ are equivalent to transformations with real parameter $\eta$ followed by a phase transformation for fermions:

$$
\left\{\Xi^{a}, \Sigma_{A}, \Sigma_{B}, \Psi_{(q)}\right\} \rightarrow e^{i \alpha}\left\{\Xi^{a}, \Sigma_{A}, \Sigma_{B}, \Psi_{(q)}\right\} .
$$

Then, $N=2$ supersymmetry requires invariance under this fermion rotation. One can easily see from Eq. (15) that fermion phase rotation invariance is achieved if and only if

$$
\lambda_{3}=\frac{g^{2}}{8}, \quad \lambda_{1}^{(q)}=\frac{\alpha_{(q)}^{2}}{8}, \quad \text { and } \quad \lambda_{2}^{(q)}=\frac{\beta_{(q)}^{2}}{8} .
$$

That is, the model is invariant under an extended supersymmetry provided relations (21) are imposed. This kind of condition appears in general when, starting from an $N=1$ supersymmetric gauge model, one attempts to impose a second supersymmetry: Conditions on coupling constants have to be imposed so as to accommodate different $N=1$ multiplets into an $N=2$ multiplet. We note that the same conditions take place in the model studied in Refs. [6,17]. Moreover, once Eqs. (21) are imposed, the Higgs potential of our model happens to be a simple generalization of that obtained in [6] by a different approach. In our case, however, it has been dictated just by supersymmetry considerations. As can be seen in Ref. [16], this discussion is analogous to that in the Abelian Higgs model.

Summarizing, we have arrived to the following $N=2$ supersymmetric action associated to the $\mathrm{SU}(2) \times \mathrm{U}(1)_{Y}$ $\times \mathrm{U}(1)_{Y^{\prime}}$ model of our interest:

$$
\begin{aligned}
\mathcal{S}_{N=2}= & \frac{1}{2} \int d^{3} x\left[-\frac{1}{2} W_{\mu \nu}^{a} W^{\mu \nu a}-\frac{1}{2} F_{\mu \nu} F^{\mu \nu}-\frac{1}{2} G_{\mu \nu} G^{\mu \nu}+\left|\mathcal{D}_{\mu}^{(1)} \Phi_{(1)}\right|^{2}+\left|\mathcal{D}_{\mu}^{(2)} \Phi_{(2)}\right|^{2}+\left(\partial_{\mu} A\right)^{2}+\left(\partial_{\mu} B\right)^{2}+\left(D_{\mu} \vec{W}\right)^{2}\right. \\
& -2 V\left(\Phi_{(1)}, \Phi_{(2)}, A, B, \vec{W}\right)+i \sum_{q=1}^{2} \bar{\Psi}_{(q)} \mathbb{D}^{(q)} \Psi_{(q)}+i \bar{\Xi}^{a}(\not D \Xi)^{a}+i \bar{\Sigma}_{A} b \Sigma_{A}+i \bar{\Sigma}_{B} b \Sigma_{B}-g f^{a b c} W^{a}\left(i \overline{\Xi^{b}} \Xi^{c}+\text { H.c. }\right) \\
& -\sum_{q=1}^{2}\left[\bar{\Psi}_{(q)}\left(\alpha_{(q)} A+\beta_{(q)} B+g W^{a} \tau^{a}\right) \Psi_{(q)}-g\left(\bar{\Psi}_{(q)} \Xi^{a} \tau^{a} \Phi_{(q)}+\text { H.c. }\right)-\alpha_{(q)}\left(\bar{\Psi}_{(q)} \Sigma_{A} \Phi_{(q)}+\text { H.c. }\right)\right. \\
& \left.\left.-\beta_{(q)}\left(\bar{\Psi}_{(q)} \Sigma_{B} \Phi_{(q)}+\text { H.c. }\right)\right]\right] .
\end{aligned}
$$

In the next section, the reasons why the conditions (21), which ensure $N=2$ supersymmetry, are also needed for the Bogomol'nyi bound will be clear in the light of the supercharge algebra. 


\section{SUPERCHARGE ALGEBRA AND BOGOMOL'NYI EQUATIONS}

We shall now analyze the $N=2$ algebra of supercharges for our model. To construct these charges we follow the Noether method. The conserved current associated with $N=2$ supersymmetry is given by

$$
\mathcal{J}_{N=2}^{\mu}=\sum_{\{\Phi\}} \frac{\delta L}{\delta \partial_{\mu} \Phi} \delta_{\eta_{c}} \Phi+\sum_{\{\Psi\}} \frac{\delta L}{\delta \partial_{\mu} \Psi} \delta_{\eta_{c}} \Psi-\theta^{\mu}\left[\eta_{c}\right]
$$

where $\{\Phi\}$ and $\{\Psi\}$ represent the whole set of bosonic and fermionic fields, respectively. Concerning $\theta^{\mu}\left[\eta_{c}\right]$, it is defined through

$$
\delta_{\eta_{c}} S=\int d^{3} x \partial_{\mu} \theta^{\mu}\left[\eta_{c}\right]
$$

The conserved charge is obtained from the current (23) as

$$
\mathcal{Q}\left[\eta_{c}\right]=\int d^{2} x \mathcal{J}_{N=2}^{0},
$$

this giving the explicit expression

$$
\begin{aligned}
\mathcal{Q}\left[\eta_{c}\right]= & -\frac{i}{2} \int d^{2} x\left\{\Sigma_{A}^{\dagger}\left[{ }^{\star} F^{\lambda} \gamma_{\lambda}+\sum_{q=1}^{2} \frac{\alpha_{(q)}}{2} \Phi_{(q)}^{\dagger} \Phi_{(q)}-\xi_{1}+i b A\right]+\Sigma_{B}^{\dagger}\left[{ }^{\star} G^{\lambda} \gamma_{\lambda}+\sum_{q=1}^{2} \frac{\beta_{(q)}}{2} \Phi_{(q)}^{\dagger} \Phi_{(q)}-\xi_{2}+i b B\right]+\Xi^{\dagger a}\left[{ }^{\star} W^{\lambda a} \gamma_{\lambda}\right.\right. \\
& \left.\left.+\frac{g}{2} \sum_{q=1}^{2} \Phi_{(q)}^{\dagger} \tau^{a} \Phi_{(q)}+i(D D W)^{a}\right]+\sum_{q=1}^{2} \Psi_{(q)}^{\dagger}\left[-i \gamma^{\mu} \mathcal{D}_{\mu}^{(q)} \Phi_{(q)}-\left(\alpha_{(q)} A+\beta_{(q)} B+g W^{a} \tau^{a}\right) \Phi_{(q)}\right]\right\} \eta_{c} .
\end{aligned}
$$

Since we are interested in connecting the $N=2$ supercharge algebra with the Bogomol'nyi relationships, we assume static configurations with $A_{0}=B_{0}=W_{0}^{a}=0$, and we restrict ourselves to a purely bosonic solution of the theory after computing the algebra. We obtain, after some calculations

$$
\left\{\overline{\mathcal{Q}}\left[\eta_{c}\right], \mathcal{Q}\left[\eta_{c}\right]\right\} \mid=2 \overline{\eta_{c}} \gamma_{0} \eta_{c} P^{0}+\overline{\eta_{c}} \eta_{c} Z,
$$

where

$$
P^{0}=E=\frac{1}{2} \int d^{2} x\left[\frac{1}{2}\left(W_{i j}^{a}\right)^{2}+\frac{1}{2}\left(F_{i j}\right)^{2}+\frac{1}{2} G_{i j}^{2}+\sum_{q=1}^{2}\left|\mathcal{D}_{i}^{(q)} \Phi_{(q)}\right|^{2}+\left(\partial_{i} A\right)^{2}+\left(\partial_{i} B\right)^{2}+\left(D_{i} \vec{W}\right)^{2}+V\left(\Phi_{(1)}, \Phi_{(2)}, A, B, W^{a}\right)\right],
$$

while the central charge is given by

$$
\begin{aligned}
Z= & -\int d^{2} x\left[\frac{1}{2} \epsilon^{i j} F_{i j}\left(\sum_{q=1}^{2} \frac{\alpha_{(q)}}{2} \Phi_{(q)}^{\dagger} \Phi_{(q)}-\xi_{1}\right)+\frac{1}{2} \epsilon^{i j} G_{i j}\left(\sum_{q=1}^{2} \frac{\beta_{(q)}}{2} \Phi_{(q)}^{\dagger} \Phi_{(q)}-\xi_{2}\right)+\frac{g}{4} \epsilon^{i j} W_{i j}^{a} \sum_{q=1}^{2} \Phi_{(q)}^{\dagger} \tau^{a} \Phi_{(q)}\right. \\
& \left.+i \epsilon^{i j} \sum_{q=1}^{2}\left(\mathcal{D}_{i}^{(q)} \Phi_{(q)}\right)\left(\mathcal{D}_{j}^{(q)} \Phi_{(q)}\right)^{*}\right] .
\end{aligned}
$$

In Eqs. (28) and (29) conditions (21) have been already imposed.

Finite energy configurations require the following asymptotic conditions on the fields,

$$
W_{i j}{ }^{a}, F_{i j}, G_{i j}, \partial_{i} A, \partial_{i} B, D_{i} W^{a}, \mathcal{D}_{i}^{(q)} \Phi_{(q)} \rightarrow 0,
$$

whereas the Higgs doublets as well as the scalar fields must minimize the potential at infinity:

$$
V\left(\Phi_{(1) \infty}, \Phi_{(2) \infty}, A_{\infty}, B_{\infty}, W_{\infty}^{a}\right)=0 .
$$

This last equation can be shown to give the following asymptotic behavior for the Higgs doublets,

$$
\Phi_{(1) \infty}=\frac{\phi_{0}}{\sqrt{2}}\left(\begin{array}{c}
0 \\
\operatorname{expin}_{(1)} \varphi
\end{array}\right), \quad \Phi_{(2) \infty}=\frac{\phi_{0}}{\sqrt{2}}\left(\begin{array}{c}
\operatorname{expin}_{(2)} \varphi \\
0
\end{array}\right)
$$

and, at the same time, the scalar fields must solve

$$
\left(\alpha_{(q)} A_{\infty}+\beta_{(q)} B_{\infty}+g W_{\infty}^{a} \tau^{a}\right) \Phi_{(q) \infty}=0 .
$$

The last term of Eq. (30) leads to expressions for $n_{(1)}$ and $n_{(2)}$ given by 


$$
n_{(1)}=-\frac{1}{2}\left(g W_{\varphi}^{3}+\alpha_{(1)} A_{\varphi}+\beta_{(1)} B_{\varphi}\right)
$$

and

$$
n_{(2)}=\frac{1}{2}\left(g W_{\varphi}^{3}-\alpha_{(2)} A_{\varphi}-\beta_{(2)} B_{\varphi}\right) \text {, }
$$

such that

$$
m \equiv n_{(1)}+n_{(2)}=-\frac{1}{2}\left(\alpha_{(1)}+\alpha_{(2)}\right) A_{\varphi}-\frac{1}{2}\left(\beta_{(1)}+\beta_{(2)}\right) B_{\varphi}
$$

is an integer which is inmediately identified as the topological charge of the configuration.

Coming back to Eq. (29) for the central charge, it can be rewritten in the form

$$
Z=\frac{1}{2} \int \partial_{i} \mathcal{V}^{i} d^{2} x
$$

where $\mathcal{V}^{i}$ is given by

$$
\mathcal{V}^{i}=\left(\xi_{1} A_{j}+\xi_{2} B_{j}+i \sum_{q=1}^{2} \Phi_{(q)}^{\dagger} \mathcal{D}_{j}^{(q)} \Phi_{(q)}\right) \epsilon^{i j}
$$

so that, after using Stokes' theorem [and taking into account the asymptotic behaviors given in Eq. (30)], we obtain

$$
Z=\oint\left(\xi_{1} A_{i}+\xi_{2} B_{i}\right) d x^{i}=-2 \pi \phi_{0}^{2} m
$$

that is, the central charge of the $N=2$ algebra equals (modulo some normalization factors) the topological charge of the configuration. This is one of the main points of our work: Once the relation between the central charge and the topological charge is established, a Bogomol'nyi bound can be easily obtained from the supersymmetry algebra [13-16].

This sort of identity between the $N=2$ central charge and topological charge was first obtained by Witten and Olive [13] in the $\mathrm{SO}(3)$ Georgi-Glashow model. It was also discussed for the self-dual Chern-Simons system by Lee, Lee, and Weinberg [14]. Hlousek and Spector [15] have thoroughly analyzed this connection by studying several models where the existence of an $N=1$ supersymmetry and a topological current implies an $N=2$ supersymmetry with its central charge coinciding with the topological charge. More recently, this connection was established for the Abelian Higgs model [16] where a condition on the coupling constants has also been shown to be necessarily imposed. This condition is unavoidable both for having $N=2$ supersymmmetry and the Bogomol'nyi equations. Also, in the study of self-dual Chern-Simons systems, having a topological charge (related to the magnetic flux) and an $N=1$ extension, a condition on the symmetry-breaking coupling constant must be imposed both to achieve $N=2$ extended supersymmetry and to obtain the Bogomolnyi equations [14].

Coming back to our model, it is now easy to find the Bogomol'nyi bound from the corresponding supersymmetry algebra. Indeed, since the brackets given by Eq. (27) can be written as a sum of fermionic bilinears,

$$
\begin{aligned}
\left\{\overline{\mathcal{Q}}\left[\eta_{c}\right], \mathcal{Q}\left[\eta_{c}\right]\right\} \mid= & \int d^{2} x\left[\left(\delta_{\eta_{c}} \Xi^{a}\right)^{\dagger}\left(\delta_{\eta_{c}} \Xi^{a}\right)\right. \\
& +\left(\delta_{\eta_{c}} \Sigma_{A}\right)^{\dagger}\left(\delta_{\eta_{c}} \Sigma_{A}\right)+\left(\delta_{\eta_{c}} \Sigma_{B}\right)^{\dagger}\left(\delta_{\eta_{c}} \Sigma_{B}\right) \\
& \left.+\sum_{q=1}^{2}\left(\delta_{\eta_{c}} \Psi_{(q)}\right)^{\dagger}\left(\delta_{\eta_{c}} \Psi_{(q)}\right)\right]
\end{aligned}
$$

it is immediate that

$$
\left\{\overline{\mathcal{Q}}\left[\eta_{c}\right], \mathcal{Q}\left[\eta_{c}\right]\right\} \mid \geqslant 0
$$

This lower bound is saturated if and only if

$$
\delta_{\eta_{c}} \Xi^{a}=\delta_{\eta_{c}} \Sigma_{A}=\delta_{\eta_{c}} \Sigma_{B}=\delta_{\eta_{c}} \Psi_{(q)}=0 .
$$

In order to further analyze the solutions of Eqs. (41), let us write the parameter $\eta_{c}$ as

$$
\eta_{c} \equiv\left(\begin{array}{c}
\eta_{+} \\
\eta_{-}
\end{array}\right)
$$

It is now easy to see that to obtain nontrivial solutions to Eqs. (41) we are forced to choose a parameter with definite chirality. Moreover, one can see that the conditions

$$
\delta_{\eta_{+}} \Xi^{a}=\delta_{\eta_{+}} \Sigma_{A}=\delta_{\eta_{+}} \Sigma_{B}=\delta_{\eta_{+}} \Psi_{(q)}=0
$$

imply $\delta_{\eta_{-}} \Xi^{a} \neq 0, \delta_{\eta_{-}} \Sigma_{A} \neq 0, \delta_{\eta_{-}} \Sigma_{B} \neq 0$, and $\delta_{\eta_{-}} \Psi_{(q)} \neq 0$ for nontrivial solutions. Hence, if one is to look for Bogomol'nyi equations corresponding to nontrivial configurations, it makes sense to consider that $\eta_{c}$ has just one independent chiral component, say,

$$
\eta_{c} \equiv\left(\begin{array}{c}
\eta_{+} \\
0
\end{array}\right)
$$

Let us note, at this point, that for a parameter of this form, the supercharge algebra can be seen to be

$$
\left\{\overline{\mathcal{Q}}\left[\eta_{+}\right], \mathcal{Q}\left[\eta_{+}\right]\right\} \mid=\eta_{+}^{\dagger} \eta_{+}\left(2 P^{0}+Z\right),
$$

with $Z$ the central charge whose explicit value is given in Eq. (38). Then, the inequality (40) is nothing but the Bogomol'nyi bound of our model:

$$
M \geqslant \pi \phi_{0}^{2} m
$$

Consequently, Eqs. (41) are the Bogomol'nyi equations of the theory (once we identify the supersymmetry parameter with $\left.\eta_{+}\right)$. Explicitly,

$$
\begin{gathered}
\epsilon^{i j} W_{i j}{ }^{a}+g \sum_{q=1}^{2} \Phi_{(q)}^{\dagger} \tau^{a} \Phi_{(q)}=0, \quad\left(D_{i} W-i \epsilon_{i j} D_{j} W\right)^{a}=0 \\
\frac{1}{2} \epsilon^{i j} F_{i j}+\sum_{q=1}^{2} \frac{\alpha_{(q)}}{2} \Phi_{(q)}^{\dagger} \Phi_{(q)}-\xi_{1}=0, \quad\left(\partial_{i}-i \epsilon_{i j} \partial_{j}\right) A=0
\end{gathered}
$$




$$
\begin{gathered}
\frac{1}{2} \epsilon^{i j} G_{i j}+\sum_{q=1}^{2} \frac{\beta_{(q)}}{2} \Phi_{(q)}^{\dagger} \Phi_{(q)}-\xi_{2}=0, \quad\left(\partial_{i}-i \epsilon_{i j} \partial_{j}\right) B=0 \\
\left(\mathcal{D}_{i}^{(q)}-i \epsilon_{i j} \mathcal{D}_{j}^{(q)}\right) \Phi_{(q)}=0 \\
\left(\alpha_{(q)} A+\beta_{(q)} B+g W^{a} \tau^{a}\right) \Phi_{(q)}=0
\end{gathered}
$$

Owing to Eq. (46), their solutions also solve the static EulerLagrange equations of motion.

Let us remark on the fact that field configurations solving the Bogomol'nyi equations break half of the supersymmetries, a feature common to all models presenting Bogomol'nyi bounds with supersymmetric extension (see, for example, [17] and references therein). Indeed, as was seen above, supersymmetry transformations generated by the antichiral parameter $\eta_{-}$are broken. If we attempt to keep all the supersymmetries of our model, we will find that the resulting field configuration has zero energy (the trivial vacuum) as easily seen from Eqs. (41). Had we been faced with an antichiral parameter in Eq. (44), we would have obtained antisoliton solutions with a breaking of the supersymmetry transformation generated by $\eta_{+}$. Analogous results also hold in four-dimensional models as the one originally studied by Witten and Olive [13].

A careful analysis of the whole set of Bogomol'nyi equations makes evident that the scalar fields $A, B$, and $\vec{W}$ must vanish. In the special case in which

$$
\alpha_{(1)} \beta_{(2)}=\alpha_{(2)} \beta_{(1)},
$$

a nonvanishing solution can be found to be

$$
A=\varphi, \quad B=-\frac{\alpha_{(1)}}{\beta_{(1)}} \varphi=-\frac{\alpha_{(2)}}{\beta_{(2)}} \varphi, \quad W^{a}=0,
$$

with $\varphi$ a real parameter describing an infinite set of vacua that, nevertheless, are physically equivalent (thus, $\varphi=0$ does not imply any loose of generality). Strikingly, we see that the configurations that saturate the Bogomol'nyi bound are exactly the same as those found in Ref. [6], in spite of the fact that our model is an extension of the electroweak theory analyzed there. Let us finally mention that, starting from Eqs. (47)-(50), it is possible to decouple an equation involving only the Higgs doublet in the same vein as it was previously done in the abelian Higgs model [12].

\section{LIMITING CASES}

As a by-product of our systematic approach, we can easily obtain Bogomol'nyi bounds (coming from an underlying $N=2$ supersymmetric structure) for a variety of models which have recently acquired physical interest.

\section{A. $\mathrm{SU}(2) \times \mathrm{U}(1)_{Y} \times \mathrm{U}(1)_{Y^{\prime}}$ pure-Higgs model}

The dynamics of this model, first considered in Ref. [6], is dictated by the following Lagrangian density:

$$
\begin{aligned}
\mathcal{L}_{p H}= & -\frac{1}{4} \vec{W}_{\mu \nu} \cdot \vec{W}^{\mu \nu}-\frac{1}{4} F_{\mu \nu} F^{\mu \nu}-\frac{1}{4} G_{\mu \nu} G^{\mu \nu} \\
& +\frac{1}{2} \sum_{q=1}^{2}\left|\mathcal{D}_{\mu}^{(q)} \Phi_{(q)}\right|^{2}-\lambda_{3}\left(\sum_{q=1}^{2} \Phi_{(q)}^{\dagger} \tau^{a} \Phi_{(q)}\right)^{2} \\
& -\left(\sum_{q=1}^{2} \sqrt{\lambda_{1}^{(q)}} \Phi_{(q)}^{\dagger} \Phi_{(q)}-\frac{\xi_{1}}{\sqrt{2}}\right)^{2} \\
& -\left(\sum_{q=1}^{2} \sqrt{\lambda_{2}^{(q)}} \Phi_{(q)}^{\dagger} \Phi_{(q)}-\frac{\xi_{2}}{\sqrt{2}}\right)^{2} .
\end{aligned}
$$

It is immediately seen that the results given in Ref. [6] can be obtained just by considering bosonic configurations satisfying the constraint

$$
A=B=W^{a}=0
$$

in our equations. ${ }^{1}$ These conditions are consistent with the asymptotic behaviors (30) and (31) and with the Bogomol'nyi equations (47)-(50) of our model. It is interesting to note that conditions (21), imposed by the requirement of extended supersymmetry, also fix in this case the coupling constants exactly as they appear in the abovementioned reference. Thus, we have shown that the potential and the coupling constants of the $\mathrm{SU}(2) \times \mathrm{U}(1)_{Y} \times \mathrm{U}(1)_{Y^{\prime}}$ pure Higgs model studied in [6] are simply dictated by $N=2$ supersymmetry. A simple ansatz for stringlike solutions of arbitrary topological charge in this system has been explored in [6]. It is shown there that, interestingly enough, these configurations do not correspond to an embedding of the Nielsen-Olesen vortex solution.

\section{B. $U(1) \times U(1)$ model}

It is well known that superconducting cosmic strings could have appeared as topological defects in the early Universe, owing to the presence of a charged field condensate in the core of the string [18]. The superconducting string models are commonly based on a $U(1) \times U(1)$ gauge symmetry $[10,18]$, where one of the $U(1)$ factors is unbroken. The same gauge group has been recently considered in order to construct the so-called binary cosmic string models [9]. However, in these models, the $U(1) \times U(1)$ symmetry is completely broken in the Higgs vacuum. In view of this, we will consider the following Lagrangian density:

$$
\begin{aligned}
\mathcal{L}_{\mathrm{SCS}}= & -\frac{1}{4} F_{\mu \nu} F^{\mu \nu}-\frac{1}{4} G_{\mu \nu} G^{\mu \nu}+\frac{1}{2}\left(D_{\mu}^{(A)} \phi\right) *\left(D^{\mu(A)} \phi\right) \\
& +\frac{1}{2}\left(D_{\mu}^{(B)} \xi\right)^{*}\left(D^{\mu(B)} \xi\right)-V(\phi, \xi)
\end{aligned}
$$

which can be obtained as a limiting case of our $\mathrm{SU}(2) \times \mathrm{U}(1)_{Y} \times \mathrm{U}(1)_{Y^{\prime}}$ system, where $\phi$ and $\xi$ are Abelian (complex) Higgs fields, while $D_{\mu}^{(A)}$ and $D_{\mu}^{(B)}$ are the covariant derivatives with respect to $A_{\mu}$ and $B_{\mu}$, respectively. In order to simplify our discussion, we will restrict ourselves to those solutions of the model satisfying condition (53), which

\footnotetext{
${ }^{1}$ Note that our algebraic approach is not modified by any constraint imposed on purely bosonic configurations, as all the fermion fields are put to zero after computing the algebra.
} 
are decoupled from the non-Abelian gauge field $W_{\mu}^{a}$. That is, we are interested in topological classical field configurations in the region of the parameter space where $g \rightarrow 0$. We then ask for solutions where the disconnected non-Abelian field strength is constrained to vanish:

$$
W_{\mu \nu}^{a}=0 .
$$

Now, we make the following ansatz for the Higgs sector:

$$
\Phi_{(1)}=\left(\begin{array}{l}
\phi \\
0
\end{array}\right) \quad \text { and } \Phi_{(2)}=\left(\begin{array}{l}
0 \\
\xi
\end{array}\right),
$$

after which the Higgs potential (10) takes the form

$$
\begin{aligned}
V(\phi, \xi)= & \lambda_{1}\left(|\phi|^{2}-v_{1}^{2}\right)^{2}+\lambda_{2}\left(|\xi|^{2}-v_{2}^{2}\right)^{2} \\
& +\lambda_{3}\left(|\phi|^{2}-v_{1}^{2}\right)\left(|\xi|^{2}-v_{2}^{2}\right) .
\end{aligned}
$$

It is worthwhile to point out that this potential satisfies the conditions for the existence of binary strings [9]. We will further simplify the system imposing the condition

$$
\alpha_{(2)}=\beta_{(1)}=0,
$$

which is equivalent to a vanishing $\lambda_{3}$ in Eq. (57), such that the effective Lagrangian looks exactly as Eq. (54), with the Higgs potential

$$
V(\phi, \xi)=\frac{\alpha_{(1)}^{2}}{8}\left(|\phi|^{2}-v_{1}^{2}\right)^{2}+\frac{\beta_{(2)}^{2}}{8}\left(|\xi|^{2}-v_{2}^{2}\right)^{2} .
$$

With this Higgs potential, the $\mathrm{U}(1) \times \mathrm{U}(1)$ symmetry is spontaneously broken. The manifold of vacua has nontrivial topology, $\Pi_{1}[\mathrm{U}(1) \times \mathrm{U}(1)]=Z \times Z$, this implying the existence of two kinds of strings: those with a flux of $A_{\mu}$ and those with a flux of $B_{\mu}$, which can be called type-A and type-B vortices, respectively. If we consider the possibility that both $\mathrm{U}(1)$ symmetries be broken roughly at the same scale, finite energy leads to the following asymptotic behavior for the Higgs fields:

$$
\phi \rightarrow v_{1} e^{i n_{Y} \theta} \quad \text { and } \xi \rightarrow v_{2} e^{i n_{Y^{\prime}} \theta},
$$

where $n_{Y}$ and $n_{Y^{\prime}}$ are integers that characterize the topological sector of $Z \times Z$ to which the solution belongs. Then, in view of Eqs. (36) and (37), it is immediately clear that the central charge of the corresponding $N=2$ supersymmetric theory becomes

$$
\widetilde{Z}=-2 \pi\left(v_{1}^{2} n_{Y}+v_{2}^{2} n_{Y^{\prime}}\right) .
$$

Thus, the Bogomol'nyi bound of the $\mathrm{U}(1) \times \mathrm{U}(1)$ model presented above is

$$
M \geqslant \pi\left(v_{1}^{2} n_{Y}+v_{2}^{2} n_{Y^{\prime}}\right) .
$$

The bound is proportional to a linear combination of the topological charges that appear do to the fact that both $\mathrm{U}(1)$ subgroups have been broken. To end this paragraph, let us comment that provided one relax any of the conditions (53) or (58), imposed in order to arrive to our quite simplified system, one could obtain Bogomol'nyi relationships for a variety of models. This analysis will be carried out elsewhere.

\section{C. $\mathrm{SU}(2)_{\text {global }} \times \mathrm{U}(1)_{\text {local }}$ semilocal model}

Finally, it is also interesting to explore how $N=2$ supersymmetry guarantees the existence of a Bogomol'nyi bound for the neutral semilocal string defects with $\mathrm{SU}(2)_{\text {global }} \times \mathrm{U}(1)_{\text {local }}$ symmetry discussed in Ref. [19], even though the vacuum manifold is simply connected. The Lagrangian density of this model takes the form

$$
\begin{aligned}
\mathcal{L}_{\mathrm{SL}}= & -\frac{1}{4} F_{\mu \nu} F^{\mu \nu}+\frac{1}{2}\left[\left(\partial_{\mu}-i e A_{\mu}\right) \Phi\right]^{\dagger}\left[\left(\partial^{\mu}-i e A^{\mu}\right) \Phi\right] \\
& -\lambda\left(\Phi^{\dagger} \Phi-v^{2}\right)^{2},
\end{aligned}
$$

where $\Phi$ is a Higgs doublet charged only under the Abelian subgroup $\mathrm{U}(1)_{\text {local }}$. The potential is minimum when $\Phi^{\dagger} \Phi=v^{2}$. Since $\Phi$ is a complex doublet, the minimum of the potential is a three-sphere and is simply connected. This is in contrast with the situation in the Abelian Higgs model where the potential minimum is a circle and a vortex solution correponds to a configuration which winds around the circle. However, it was explicitly shown in Ref. [19] that this model admits of stable string solutions by a simple generalization of Bogomol'nyi's proof. We can reproduce their proof as a particular case of our model. In fact, it is easy to see that imposing conditions (53) and (55), and working in the parameter space region where $g, \beta_{(q)} \rightarrow 0$, we just have to restrict ourselves to those configurations satisfying the constraint

$$
\Phi_{(2)}=G_{\mu \nu}=0 .
$$

Then, the Bogomol'nyi bound obtained in [19] can be easily reproduced following the same steps as above. Let us mention that the Bogomol'nyi bound survives the coupling of this system to the gravitational field [20]. It is also possible, in this case, to show that this bound can be thought of as coming from an underlying supergravity model [21].

Let us end our paper remarking that we have considered a $\mathrm{SU}(2) \times \mathrm{U}(1)_{Y} \times \mathrm{U}(1)_{Y^{\prime}}$ gauge model with a symmetrybreaking potential, which can be seen to be a simple extension of the electroweak standard model. The requirement of $N=2$ supersymmetry forces a relation between coupling constants and at the same time, through its supercharge algebra, imposes the Bogomol'nyi equations on certain classical field configurations. The connection of our model with realistic supersymmetric extensions of the standard model and the possible existence of stringlike solutions in its coupling to supergravity remain open problems. We hope to report on these issues in a forthcoming work.

\section{ACKNOWLEDGMENTS}

This work was partially supported by CONICET, Argentina. We would like to thank F. Schaposnik for a critical reading of the manuscript. J.D.E. is pleased to thank the Departament d'Estructura i Constituents de la Materia for its kind hospitality. 
[1] G. G. Ross, Grand Unified Theories, Frontiers in Physics Vol. 60 (Benjamin-Cummings, New York, 1984).

[2] Particle Data Group, L. Montanet et al., Phys. Rev. D 50, 1173 (1994).

[3] See, for example, P. Nath, R. Arnowitt, and A. H. Chamseddine, Applied $N=1$ Supergravity, ICTP Series on Theoretical Physics, Vol. 1 (World Scientific, Singapore, 1984), and references therein.

[4] G. Dvali and G. Senjanovich, Phys. Rev. Lett. 71, 2376 (1993); L. Perivolaropoulos, Phys. Lett. B 316, 528 (1993); M. A. Earnshaw and M. James, Phys. Rev. D 48, 5818 (1993); T. N. Tomaras, in Search for Solitons in Two-Higgs Extensions of the Standard Model, Proceedings of the Conference "Topics in Quantum Field Theory: Modern Methods in Fundamental Physics,' Maynooth, Ireland, 1995, edited by D. H. Tchrakian (World Scientific, Singapore, 1995); C. Bachas and T. N. Tomaras, Phys. Rev. Lett. 76, 356 (1996).

[5] M. Goodband and M. Hindmarsh, Phys. Lett. B 370, 29 (1996).

[6] G. Bimonte and G. Lozano, Phys. Lett. B 326, 270 (1994).

[7] H. B. Nielsen and P. Olesen, Nucl. Phys. B61, 45 (1973).

[8] P. Peter, Phys. Rev. D 49, 5052 (1994); A-C. Davies and P.
Peter, Phys. Lett. B 358, 197 (1995).

[9] R. Rohm and I. Dasgupta, Phys. Rev. D 53, 1827 (1996).

[10] J. R. Morris, Phys. Rev. D 53, 2078 (1996).

[11] E. B. Bogomol'nyi, Sov. J. Nucl. Phys. 24, 449 (1976).

[12] H. de Vega and F. Schaposnik, Phys. Rev. D 14, 1100 (1976).

[13] E. Witten and D. Olive, Phys. Lett. 78B, 97 (1978).

[14] C. Lee, K. Lee, and E. J. Weinberg, Phys. Lett. B 243, 105 (1990).

[15] Z. Hlousek and D. Spector, Nucl. Phys. B370, 143 (1992); B397, 173 (1993); Phys. Lett. B 283, 75 (1992); Mod. Phys. Lett. A 7, 3403 (1992).

[16] J. D. Edelstein, C. Núñez, and F. A. Schaposnik, Phys. Lett. B 329, 39 (1994).

[17] J. D. Edelstein, C. Núñez, and F. A. Schaposnik, Nucl. Phys. B458, 165 (1996).

[18] E. Witten, Nucl. Phys. B249, 557 (1985).

[19] T. Vachaspati and A. Achúcarro, Phys. Rev. D 44, 3067 (1991).

[20] G. W. Gibbons, M. E. Ortiz, F. Ruiz Ruiz, and T. M. Samols, Nucl. Phys. B385, 127 (1992).

[21] J. D. Edelstein, Phys. Lett. B 390, 101 (1997). 